\title{
Getting to the Core of the Women's Curriculum-It's Time to Change the Way We Train Our Fellows
}

\author{
Deborah D. Proctor
}

Published online: 21 January 2011

(C) Springer Science+Business Media, LLC 2011

In the United States, 50.7 percent of the population are women [1]. In 2004 there were an estimated 104 million visits for evaluation and management of a digestive disease, and after adjustment for age the number of visits was 10 percent higher for women than for men, which equates to over 57 million visits per year for women [2]. Although many digestive diseases are the same in men and women, there are also many differences. Research has shown that there are gender differences in epidemiology, response to health and illness, treatment, and complications. Fertility, pregnancy, and the post-partum state pose specific challenges for gastroenterologists. As specialists in digestive diseases, gastroenterologists must have the knowledge and range of skills necessary to care for women with their unique issues.

It is challenging to successfully train highly qualified gastroenterologists during the three year fellowship period. An enormous amount of knowledge and procedural skills must be acquired by the end of training, with the instillation of a desire for life-long learning. A program must be well-structured with a committed program director and dedicated faculty in order to accomplish these objectives.

Guidelines for training gastroenterology fellows were first published by the Gastroenterology Leadership Council in 1996, but it was not until the Gastroenterology Core Curriculum was updated in 2003 that a women's curriculum in digestive diseases was first outlined. The recommendations specifically addressed three areas: (1) general women's gastroenterology (GI) health issues, including the doctorpatient relationship; cultural, religious, and psychosocial

D. D. Proctor $(\bowtie)$

Yale University, New Haven, CT, USA

e-mail: deborah.proctor@yale.edu issues; differences in disease presentation; laboratory and diagnostic testing; and the use of complementary and alternative medicine; (2) specific women's GI health issues including gender differences in GI health and disease states, the effects of the menstrual cycle and menopause on GI health and disease states, medication pharmacokinetics, and response to and complications of therapy; and (3) pregnancy and childbearing issues, including fertility, infertility, pregnancy in women with preexisting digestive diseases, diseases unique to pregnancy, medication pharmacokinetics and safety during pregnancy and with breast-feeding, clinical onset of digestive diseases during pregnancy and after delivery, and the effect of pregnancy on healthy mothers. The training recommendations included obtaining the appropriate knowledge and clinical experience through didactic lectures and close alliance with physicians who have an interest and experience in evaluating and managing women's GI health issues, including obstetricians and gynecologists. Another recommendation was that a minimum of $25 \%$ of the fellow's panel of patients be women [3].

In July 2005, the ACGME adopted these recommendations and now requires formal instruction and clinical experience in women's GI health issues and that in their continuity clinic "each fellow's panel of patients must include at least $25 \%$ of patients from each gender" [4].

In 2008, the first survey assessing compliance with the Gastroenterology Core Curriculum was published [5]. This study evaluated overall programmatic compliance with a focus on the hepatology component and found that although most programs were in general compliance with the recommendations of the Core Curriculum, and specifically the hepatology portion, there were striking deficiencies noted in some areas of hepatology training. Although this study was somewhat reassuring, the areas where there were shortcomings needed improvement [5]. 
In this issue of Digestive Diseases and Sciences, Dr. Saha and her colleagues [6] tackle the Gastroenterology Core Curriculum again, this time the women's training recommendations. The objectives of the study were to determine whether or not at the end of a three-year fellowship, fellows are competent to evaluate and manage women's GI health issues. If competence was in doubt, attempts were made to identify barriers to preparedness. The target population for this study was all adult GI program directors and approximately 50\% of GI fellows in training in the United States. Programs were randomly selected to have all of their fellows surveyed; the number was chosen for statistical reasons.

It was reassuring to know that program directors' and fellows' attitudes toward women's GI health issues were favorable. Using a four-point Likert scale, both program directors and fellows had favorable attitudes toward training, with fellows having statistically higher scores throughout their three years than did program directors. This suggests willingness and interest on the part of the fellows to be trained in women's GI health issues.

However, it was very disheartening to see the striking deficiencies in didactic teaching, clinical training, and, most importantly, the fellows' self-reported lack of preparedness in evaluating and managing women's GI issues. Using a five-point Likert scale, both program directors and fellows perceived fellows' didactic teaching to be insufficient, with fellows feeling even more than program directors that the quantity of didactic teaching experiences was too little (mean score $=2.32$ ). Likewise using a five-point Likert scale, both program directors and fellows reported clinical experiences and training in women's GI health issues were infrequent with, once again, fellows reporting even fewer experiences than program directors (mean score $=2.58$ ). Last, and perhaps most damaging, fellow and program director ratings for trainees' level of preparedness in the evaluation and management of women's GI health issues was far less than what the public expects of physicians. Fellows rated themselves as being less than "somewhat prepared" to evaluate and manage women's GI health issues, self-reporting which was present even for fellows who were approaching graduation.

Some of the barriers to achieving the objectives of competence in evaluating and managing women's GI health issues are obvious, but will be difficult to fix. For example, $44.5 \%$ of program directors reported that some or all of their fellows fulfilled the mandatory half day per week ambulatory care clinic requirement at a Veterans Administration (VA) hospital. Even with more and more women serving in the military, the VA population is still less than $25 \%$ women. However, programs that use VA hospitals as clinical training sites have financial arrangements with them which will be difficult, if not impossible, to replace. A compromise position would be to add a second non-VA clinic while continuing the VA clinic.

Other barriers should be easier to overcome, but will still require cooperation and compromise. For example although $86 \%$ of programs reported obstetric services at their programs, almost $25 \%$ of fellows reported no (zero!) consultations from this service during their fellowship training. Not surprisingly, $27 \%$ of third-year fellows reported caring for fewer than five pregnant women during their fellowship. Poor collaboration between gastroenterology and obstetric/gynecology physicians was reported by $28 \%$ of program directors. Poor collaboration should not exist between any group of physicians and must be fixed in order for our fellows to be better able to manage women's GI issues. In the programs where this exists, compromise and cooperation must be undertaken and collaboration improved. The stakes are high.

Although this study has many strengths, there are several limitations. The response rate for program directors was high (94 or $61 \%$ ), but low for fellows (228 or $30 \%$ ). This is comparable with mail survey responses of physicians in other studies [7], but does introduce the potential for non-response bias. Second, one-third of the fellows responding were first-year fellows with limited exposure. The authors adjusted for this potential limitation by taking into account the year of training. Third, the study relies on self-reporting and the authors did not verify clinical experiences or didactic teaching sessions. However, the assumption was made that program directors and fellows would be truthful, because this was a confidential survey. And, last, the authors inquired about a limited number of potential barriers to training, but did not address others such as costs of training and time to acquire training.

Despite these limitations, this study provides very important information for program directors and training programs for training gastroenterologists of the future. It is very encouraging that fellows are interested in learning about, and want to be appropriately trained in, evaluation and management of women's GI health issues. As the field of digestive diseases continues to grow and consideration of more focused areas of specialization, for example hepatology, motility, and inflammatory diseases, is in progress, training programs must integrate gender differences and similarities into all of their lectures and clinical training. Suggestions for accomplishing this include: (1) didactic lectures from faculty with experience and interest in women's issues; (2) clinical experiences for our fellows in evaluating and managing women's GI diseases-inpatient and outpatient clinics with women patients and the opportunity to perform procedures on women patients; and (3) closer collaborations with obstetric/gynecology services. As indicated in this study's survey results, this 
knowledge and experience will be embraced by our fellows and ultimately translate into better care for our patients.

\section{References}

1. US Census Bureau Facts. Available at: http://www.census.gov/ popest/national/asrh/NC-EST2009-srh.html. Accessed December 22, 2010.

2. Everhart JE. All Digestive Diseases. In: Everhart JE, ed. The burden of digestive diseases in the United States. US Department of Health and Human Services, Public Health Service, National Institutes of Health, National Institute of Diabetes and Digestive and Kidney Diseases. Washington, DC: US Government Printing Office, 2008; NIH Publication No. 09-6443:1-8.

3. Gastroenterology Leadership Council. American Association for the Study of Liver Disease, American College of Gastroenterology,
American Gastroenterological Association, and American Society for Gastointestinal Endoscopy. Training the gastroenterologist for the future: The Gastroenterology Core Curriculum. Gastroenterol. 2003;124:1055-1104.

4. ACGME Program Requirements for Graduate Medical Education in Gastroenterology. Available at: http://www.acgme.org/acWebsite/ RRC_140/140_prIndex.asp. Accessed December 20, 2010.

5. Guardino JM, Proctor DD, Lopez R, Carey W. Utilization of and adherence to the gastroenterology core curriculum on hepatology training during a gastrointestinal fellowship. Clin Gastroenterol Hepatol. 2008;6:682-688.

6. Saha S, Roberson E, Richie K, Lindstrom MJ, Esposti SD, Wald A. Women's health training in gastroenterology fellowship: a national survey of fellows and program directors. Dig Dis Sci. 2011; ADD CITATION.

7. Sudman S. Mailed survey of reluctant professionals. Eval Rev. 1985;9:349-360. 